\title{
Neutropenia febril de bajo riesgo en pacientes oncológicos
}

\author{
CLAUDIA ROJO L. ${ }^{1}$, NATALIE RODRÍGUEZ Z. ${ }^{2,3}$, JUAN TORDECILLA C.,3 \\ 1. Becada. Programa de Especialización en Pediatría. Departamento de Pediatría y Cirugía Infantil. Campus Norte. \\ Facultad de Medicina. Universidad de Chile. \\ 2. Departamento de Pediatría y Cirugía Infantil. Campus Norte. Facultad de Medicina. Universidad de Chile. \\ 3. Unidad de Hemato-Oncología. Hospital de Niños Roberto del Río.
}

\begin{abstract}
Low risk febrile neutropenia in oncological pediatric patients: clinical experience

Background: Infections constitute the major cause of morbimortality in pediatric patients treated with chemotherapy. The PINDA Infectology Committee (National Program of Antineoplasic Drugs) validated a model to predict the risk of invasive bacterial infection (IBI) in oncological patients with febrile neutropenia (FN), in order to differentiate episodes of low risk (LRFN) versus high risk (HRFN) of IBI. Objective: Characterize LRFN episodes in patients treated at the Oncology Unit of Hospital Roberto del Río, between 2003-2006. Method: Retrospective study of clinical charts of patients with cancer and LRFN in the period of time selected. Results: 185 patients with 202 FN episodes registered, where 47 correspond to LRFN (23\%). $8.5 \%$ of LRFN episodes had an unfavourable outcome (not statistically significant). In these patients, cultures were negative and required more days of hospitalization. No patients were readmitted and had no infection associated mortality. Conclusion: The selective management of patients with LRFN following the current criteria is safe and effective.

(Key words: pediatric oncological patients, invasive bacterial infection, low risk febrile neutropenia).

Rev Chil Pediatr 2008; 79 (2): 157-162
\end{abstract}

\section{RESUMEN}

Las infecciones son la principal causa de morbimortalidad en pacientes oncológicos en quimioterapia. El Comité de Infectología del PINDA (Programa Infantil Nacional de Drogas Antineoplásicas) validó un modelo de predicción de riesgo de infección bacteriana invasora (IBI) en niños oncológicos con neutropenia febril (NF), que discrimina episodios de bajo (NFBR) y alto riesgo (NFAR) de IBI. Objetivo: Caracterizar los episodios de NFBR de pacientes atendidos en la Unidad de Oncología del Hospital de Niños Roberto del Río (2003-2006). Pacientes y Métodos: Revisión retrospectiva de fichas clínicas de pacientes que debutaron con patología oncológica en ese período que presentaron episodios de NFBR consignando edad, sexo, diagnóstico oncológico de base, cateter venoso central, foco infeccioso, quimioterapia y cultivos. Se

Trabajo recibido el 02 de mayo de 2007, devuelto para corregir el 11 de julio de 2007, segunda versión el 27 de diciembre de 2007, aceptado para publicación el 23 de enero de 2008.

Correspondencia a:

Claudia Rojo L.

E-mail: claukrl@gmail.com 
evaluó la evolución clínica por parámetros clínicos y de laboratorio, y se analizaron las variables mediante prueba de $\chi^{2}$, con un p < 0,05. Resultados: En 185 pacientes, se registraron 202 episodios de NF, 47 fueron NFBR (23\%). Presentaron evolución desfavorable un 8,5\% de NFBR ( $>>0,05$ ). En ellos no se logró aislamiento microbiológico y tuvieron mayor número de días de hospitalización. No hubo en este grupo reingresos ni mortalidad asociada. Conclusión: El manejo selectivo de los pacientes con NFBR, según criterios actuales, es seguro y efectivo.

(Palabras clave: paciente oncológico pediátrico, infección bacteriana invasora, neutropenia febril de bajo riesgo).

Rev Chil Pediatr 2008; 79 (2): 157-162

\section{Introducción}

Los pacientes oncológicos pediátricos atendidos en el sistema público de salud son tratados de acuerdo a protocolos incluidos en el Programa Infantil Nacional de Drogas Antineoplásicas (PINDA) del Ministerio de Salud. Los esquemas de quimioterapia actualmente en uso han permitido mejorar la sobrevida de estos pacientes, pero su intensidad se asocia a neutropenias severas, que se prolongan por espacio de 7 días en promedio, lo que favorece la aparición de infecciones bacterianas graves, constituyendo una de las principales causas de morbimortalidad en estos pacientes ${ }^{1-3}$.

Diversos estudios nacionales e internacionales han intentado caracterizar los episodios de neutropenia febril con el fin de determinar aquellas variables que se asocian con un mayor riesgo de bacteriemia y muerte ${ }^{2-8}$.

El Comité de Infectología del PINDA entre los años 1996 y 1997 realizó un estudio multicéntrico para identificar las variables clínicas y de laboratorio, presentes al momento del ingreso del niño oncológico con neutropenia febril, que permitieran pesquisar aquellos pacientes con un alto riesgo de desarrollar una infección bacteriana invasora (IBI). Este estudio concluyó que había cinco factores involucrados: nivel de Proteína C Reactiva (PCR) $\geq$ a $90 \mathrm{mg} / \mathrm{L}$, hipotensión, recaída de leucemia, recuento de plaquetas menor o igual a $50000 \mathrm{x} \mathrm{mm}^{3} \mathrm{y}$ quimioterapia reciente (menos o igual a 7 días desde el inicio de la quimioterapia). La IBI se presentó en 2\%,17\%, 48\%,75\% y 100\% de los casos si los episodios se asociaban a ningún, 1 , 2, 3 y 4 o más factores de riesgo respectivamente. Estas variables tienen una sensibilidad, especificidad, valor predictivo positivo y valor predictivo negativo de 92, 76, 82 y 90\% respectivamente. El riesgo relativo de presentar IBI aumenta significativamente si el paciente presenta 2 o más factores de riesgo ${ }^{4,5,9}$.

El año 2000 se validó este modelo de predicción de riesgo de IBI en niños oncológicos con neutropenia febril, discriminando entre episodios de bajo y alto riesgo. Se catalogaron como episodios de neutropenia febril de alto riesgo (NFAR) aquellos que se asociaron a los factores que se detallan a continuación. Como factores únicos, la presencia de $\mathrm{PCR}>$ a $90 \mathrm{mg} / \mathrm{L}$ o hipotensión arterial o alguno de los siguientes tipos de cáncer: leucemia en recaída, leucemia mieloide aguda, linfoma no Hodgkin, neuroblastoma etapa IV y recaída de tumores sólidos. Los episodios de neutropenia febril de bajo riesgo (NFBR) corresponden a aquellos que no presentaron los factores antes descritos o que presentaron como factor único un recuento de plaquetas $<50000 \times \mathrm{mm}^{3}$ o menos de 7 días desde la última quimioterapia ${ }^{4,5,9}$.

En nuestro país, entre los años 2000 y 2003, se realizó un estudio multicéntrico, que comparó la eficacia clínica y el costo de 2 estrategias terapéuticas en 149 episodios de NFBR: tratamiento antibiótico ambulatorio versus hospitalizado, demostrando una eficacia similar de 95 y 93\% respectivamente, pero con un significativo menor costo en el caso de los pacientes tratados ambulatoriamente, además de un menor impacto psicosocial en su evolución. En los episodios de NFBR la tendencia actual es utilizar monoterapia antibiótica, priorizando el manejo ambulatorio del paciente. Las experiencias publicadas demuestran la seguridad del uso de antibióticos orales, según el foco clínico que 
presenta el paciente a su ingreso y el alta precoz, sin riesgo para el niño ${ }^{6,10-16}$.

Los estudios actuales se centran principalmente en caracterizar la evolución de los episodios de alto riesgo, debido al impacto que produce su manejo adecuado y precoz, en la sobrevida de estos pacientes. En relación a la evolución de los episodios que se catalogan como de bajo riesgo, se sabe que algunos de ellos pueden presentar una evolución desfavorable, que requiera intensificar los tratamientos y por lo tanto, en este grupo parece importante predecir que pacientes podrían comportarse de este modo y presentar riesgo de una infección severa, a pesar de haber sido catalogados como de bajo riesgo de IBI al diagnóstico ${ }^{16}$.

Nuestro objetivo fue caracterizar desde el punto de vista clínico y de laboratorio, los episodios de NFBR atendidos en la Unidad de Oncología, entre los años 2003 y 2006, y describir su evolución.

\section{Pacientes y Métodos}

\section{Selección de pacientes}

Se incluyeron todos los pacientes menores de 15 años con diagnóstico de patología oncológica atendidos en la Unidad de Oncología del Hospital Roberto del Río entre enero 2003 y mayo 2006. Se revisó en forma retrospectiva la ficha clínica de cada paciente, buscando episodios de neutropenia febril catalogados como de bajo riesgo, según los criterios planteados por el Comité de Infectología del PINDA $^{3,4}$.

\section{Evaluación inicial y manejo}

En cada uno de los episodios se consignó edad, sexo, diagnóstico oncológico de base, tipo y fecha de instalación de catéter venoso central (CVC), foco infeccioso al ingreso, ciclo de quimioterapia recibida y fecha, uso de factor de crecimiento de colonias de granulocitos (GCSF) post quimioterapia, recuento leucocitario y recuento absoluto de neutrófilos (RAN), cifra de plaquetas, proteína $\mathrm{C}$ reactiva (PCR) y resultados de cultivos (hemocultivos periféricos y centrales, urocultivos, coprocultivos y otros).

Todos los pacientes recibieron Ceftriaxona endovenosa (100 mg/kg/día), independiente del foco infeccioso detectado clínicamente al ingreso, previa toma de cultivos, de acuerdo a protocolo ${ }^{3,4}$.

Se realizó control clínico y de laboratorio a las 24 y 48 horas del ingreso de los pacientes, luego cada dos días hasta que el RAN fue mayor a $500 \mathrm{x} \mathrm{mm}^{3}$. Se consignó además aislamiento microbiológico, días de hospitalización, evolución de cada episodio, necesidad de atención en Unidad de Cuidados Especiales, mortalidad y reingreso al hospital posterior al alta de dicho episodio.

\section{Evolución y conducta terapéutica}

Se definió evolución favorable la estabilidad hemodinámica, curso afebril, descenso de la PCR, resolución de focos infecciosos presentes al ingreso y ausencia de nuevos focos de infección. En estos pacientes se mantuvo la terapia con Ceftriaxona endovenosa y si los hemocultivos eran negativos a las 48 horas, sin neumonía clínica o radiológica, los pacientes eran dados de alta; si los cultivos resultaban positivos se modificó la terapia antibiótica de acuerdo al agente etiológico y su sensibilidad, y en algunos casos se indicó tratamiento antibiótico oral, si se demostraba algún foco infeccioso de probable etiología bacteriana (respiratorio no neumonía, urinario y/o digestivo $)^{6}$.

Se consideró como evolución desfavorable la presencia de uno o más de los siguientes eventos:

- Aparición de inestabilidad hemodinámica no atribuible a pérdida de volumen.

- Fiebre persistente más de 72 horas del inicio de tratamiento antibiótico.

- Aparición de un nuevo foco infeccioso sugerente de IBI.

- Curva de PCR estacionaria o reducción no significativa a partir del tercer día (menor al $30 \%$ ) o en ascenso dentro de las 72 horas de iniciado el tratamiento antibiótico.

- Persistencia de cultivos positivos 72 horas post tratamiento.

Estos pacientes fueron reevaluados con exámenes de laboratorio: hemograma, PCR, hemocultivos u otros cultivos dependiendo del foco clínico. El tratamiento antibiótico se modificó 
entonces, utilizando terapia antibiótica triasociada, como en los episodios de NFAR, con Cefotaxima (150 mg/kg/día), Cloxacilina (200 $\mathrm{mg} / \mathrm{kg} /$ día) y Amikacina (15 mg/kg/día). Se mantuvo la reevaluación clínica y de laboratorio, ajustando la terapia según hallazgos microbiológicos y/o aparición de nuevos focos, incluyendo antifúngicos, si la fiebre se mantenía por más de cinco días o frente a la presencia de cultivos y/o imágenes que orientaban a posible infección por hongos.

\section{Análisis estadístico}

Las variables ordinales fueron analizadas mediante proporciones empleando $\chi^{2}$.

Las comparaciones de promedio se hicieron mediante tabla $\chi^{2}$ con corrección de Yates. Se consideraron significativas si el $\mathrm{p}$ value fue menor a 0,05 .

\section{Resultados}

Ingresaron 185 pacientes, registrándose 202 episodios de NF, de los cuales 155 fueron catalogados como NFAR y 47 como NFBR, lo que correspondió al 77 y $23 \%$ del total de episodios de NF respectivamente. De estos 47 episodios, 43 evolucionaron favorablemente según los criterios del Comité de Infectología del PINDA.

Los 43 episodios de NFBR que evolucionaron en forma favorable se presentaron en 29 pacientes, lo que representa un promedio de 1,5 episodios por paciente, con igual número de episodios en ambos sexos y con una mediana de edad de 5 años 10 meses (rango: 1 año 2 meses a 14 años 9 meses).

El diagnóstico de base correspondió en su mayoría a leucemia linfoblástica aguda en etapa de consolidación o mantención (57\%), luego tumores sólidos como: rabdomiosarcoma, tumor neuroectodérmico primitivo (PNET)/ Sarcoma de Ewing, hepatoblastoma y neuroblastoma.

En relación al foco clínico al ingreso, en orden de frecuencia, estos fueron: respiratorio, fiebre origen desconocido, gastrointestinal, oral y urinario.

Se logró determinar la etiología microbiológica en 7 de los 43 episodios. En 5 episodios se obtuvo hemocultivos positivos (centrales y periféricos), siendo los microorganismos aislados: Staphylococcus coagulasa negativo (3), Escherichia coli (1) y Streptococcus viridans (1). Los otros 2 episodios presentaron Ig M positiva para Mycoplasma pneumoniae e inmunofluorescencia indirecta para virus Influenza B. El promedio de días de hospitalización fue 5,1. En este grupo no hubo pacientes que requirieron atención en Unidad de Cuidados Especiales. Tampoco hubo reingreso al hospital en aquellos casos, en que por su evolución, se decidió alta hospitalaria precoz, ni mortalidad asociada a infección.

De los 47 episodios de NFBR hubo 4 que presentaron evolución desfavorable, los que se presentaron en 4 pacientes diferentes. Esto corresponde a una frecuencia de 8,5\% del total (p 0,43). Las características de los pacientes que presentaron evolución desfavorable son las siguientes: sexo masculino y mediana de edad 6 años 10 meses (rango: 5 años 6 meses a 9 años 6 meses). Las patologías de base más frecuentes fueron leucemia linfoblástica aguda y rabdomiosarcoma. Todos los pacientes tenían entre 12 y 24 horas de evolución del cuadro febril antes de su ingreso al hospital. Los focos clínicos encontrados al ingreso fueron oral o mucositis (2), gastrointestinal (1) y respiratorio (1). El promedio de días de hospitalización fue 7,7 y en estos 4 episodios no se logró confirmación etiológica. No hubo diferencias en cuanto a la presencia de CVC, ni al uso de G-CSF (tabla 1).

\section{Discusión}

Los episodios de neutropenia febril son una complicación frecuente en los pacientes pediátricos oncológicos, que reciben esquemas de quimioterapia cada vez más intensos. Hasta la década de los 90, el manejo de esta complicación, de alta mortalidad, se basaba en un tratamiento caracterizado por el uso de terapia antibiótica empírica, de amplio espectro, administrada en el hospital hasta la recuperación de la neutropenia ${ }^{1,2}$.

Una estrategia de "manejo selectivo" en estos pacientes, ha demostrado ser la más ade- 
Tabla 1. Características de los episodios de NFBR

\begin{tabular}{lll}
\hline $\begin{array}{c}\text { Episodios de NFBR } \\
(\mathbf{n = 4 7 )}\end{array}$ & \multicolumn{1}{c}{$\begin{array}{c}\text { Evolución favorable } \\
(\mathbf{n = 4 3 )}\end{array}$} & \multicolumn{1}{c}{$\begin{array}{c}\text { Evolución desfavorable } \\
(\mathbf{n = 4 )}\end{array}$} \\
\hline Sexo & Hombres: $19(44 \%)$ & Hombres: $4(100 \%)$ \\
& Mujeres: $24(56 \%)$ & Mujeres: 0 \\
Edad (mediana) & 5 años 10 meses & 6 años 10 meses \\
Patología de base & Leucemia aguda: $25(58 \%)$ & Leucemia aguda: $2(50 \%)$ \\
& Tumores sólidos: $18(42 \%)$ & Tumores sólidos: $2(50 \%)$ \\
Foco infeccioso al ingreso & Respiratorio: $17(40 \%)$ & Respiratorio: $1(25 \%)$ \\
& FOD: $16(37 \%)$ & FOD: 0 \\
& Gastrointestinal: $8(19 \%)$ & Gastrointestinal: $1(25 \%)$ \\
& Oral: $1(2 \%)$ & Oral: $2(50 \%)$ \\
Promedio díashospitalización & Urinario: $1(2 \%)$ & 7,7 \\
Diagnóstico microbiológico & 5,1 & $0 \%$ \\
\hline
\end{tabular}

FOD: Fiebre de origen desconocido

cuada. Esta conducta permite, por un lado, intensificar las medidas de apoyo y la terapia antibiótica en aquellos casos de alto riesgo de IBI, en donde se concentra la mayor mortalidad, y por otro lado, disminuir la toxicidad, la selección de microorganismos resistentes a antibióticos y los días de hospitalización, en aquellos casos con bajo riesgo de infección severa ${ }^{16}$.

En nuestro país, los grupos oncológicos pediátricos han adoptado tratamientos para los episodios de neutropenia febril, basados en los estudios y recomendaciones del Comité de Infectología del PINDA, diferenciando el manejo de las NF de alto y bajo riesgo de $\mathrm{IBI}^{3-6,9-11}$.

Los hallazgos de nuestro estudio descriptivo son similares a los encontrados en la literatura, respecto a la frecuencia de NFBR en pacientes oncológicos en tratamiento con quimioterapia. En general estos episodios corresponden a un tercio de los episodios de neutropenia febril. En relación a los focos de infección de los pacientes con NFBR, en nuestro estudio, a diferencia de los resultados reportados previamente, se evidencia un discreto predominio del foco respiratorio por sobre la fiebre de origen desconocido $3,4,9-11$.

$\mathrm{Al}$ analizar los episodios de NFBR que evolucionaron en forma desfavorable, destaca que la frecuencia encontrada en este estudio, es mayor a la descrita en la experiencia de Santolaya y cols: 8/149 (5,3\%) vs 4/47 (8,5\%), pero con p no significativo (p 0,43). Ello no se asoció a mayor mortalidad ni a un aumento en el traslado de estos pacientes a la Unidad de Cuidados Especiales, atribuible exclusivamente a la incapacidad del modelo de detectar pacientes de mayor riesgo de $\mathrm{IBI}^{3,4,11}$.

Ya que sólo 4 episodios presentaron una evolución desfavorable, es muy difícil plantear características destacables en este grupo que permitan diferenciarlos. Como se observa en la tabla 1, en los episodios de evolución desfavorable la mediana de edad fue discretamente mayor, 6 años 10 meses (con un rango de 5 años 6 meses y 9 años 10 meses). En cuanto al sexo, en el grupo de evolución favorable hay un discreto predominio de mujeres (24 vs 19), sin embargo, en el grupo de evolución desfavorable el $100 \%$ fueron hombres. El diagnóstico más frecuente, en los episodios de evolución favorable y desfavorable fue leucemia. Con respecto a los focos de ingreso, en el grupo de evolución favorable, la fiebre de origen desconocido (FOD) y el foco respiratorio abarcan el $70 \%$ (33 episodios), sin embargo, la FOD no se observó en los episodios con evolución desfavorable, siendo los focos de ingreso: oral (2), respiratorio (1) y gastrointestinal (1). Este grupo presenta además un aumento en el promedio de días de hospitalización, pero no hay diferencias en relación al número total de días con neutropenia severa.

En nuestro estudio observamos aislamiento 
microbiológico (positividad de hemocultivos) en el $11 \%$ de los casos en el grupo de evolución favorable (n: 43) y no logramos aislamiento microbiológico en los episodios de evolución desfavorable, lo que llama la atención, ya que esperábamos que la evolución desfavorable se asociara a la presencia de otros agentes no susceptibles al tratamiento empírico utilizado.

En base a nuestra experiencia y a la revisión de la literatura en este tema, creemos que hasta ahora, el sistema de categorización de riesgo y "manejo selectivo" de los pacientes oncológicos pediátricos con neutropenia febril, según las recomendaciones del Comité de Infectología del PINDA, ha demostrado ser efectivo y seguro.

Estos datos representan la realidad de nuestra unidad, sin embargo, pueden servir para futuros estudios que se enmarquen en la línea de desarrollo del Comité de Infectología del PINDA, lo que permita caracterizar mejor los pacientes con NFBR que tendrán una evolución desfavorable, en quienes sea necesario modificar la terapia empírica en forma más precoz.

\section{Referencias}

1.- Pizzo PA, Freifeld A, Walsh T, Alexander S: Infectious complications in pediatric cancer patients. Capítulo 41 Principles and practice of pediatric oncology,fourth edition Pizzo PA, Poplack DG 2002 Lippincott Williams \& Wilkins.

2.- Pizzo PA, Rubin M, Freifeld A, et al: The child with cancer and infection I. Empiric therapy for fever and neutropenia, and preventive strategies. J Pediatr 1991; 119: 679-94.

3.- Santolaya ME, Rabagliati $R$, Bidart T, Payá E, Guzmán AM, et al: Consenso manejo racional del paciente con cáncer, neutropenia y fiebre. Rev Chil Infect 2005; 22 (supl 2): S79-113.

4.- Santolaya ME: Neutropenia febril en el niño con cáncer. Conceptos actuales sobre criterios de riesgo y manejo selectivo. Rev Méd Chile 2001; 129: 1449-54.

5.- Santolaya ME, Álvarez AM, Avilés CL, et al:
Prospective evaluation of a model of prediction of invasive bacterial infection risk among children with cancer, fever and neutropenia. Clin Infect Dis 2002; 35: 678-83.

6.- Tordecilla J, Campbell M, Joannon P: Criterios de alta precoz en niños con cáncer y neutropenia febril. Rev Chil Pediatr 1998; 69: 247-51.

7.- Kern W: Risk assessment and risk-based therapeutic strategies in febrile neutropenia. Curr Opin Infect Dis 2001; 14: 415-22.

8.- Klastersky J, Paesmans M, Rubinstein EB, et al: The Multinational Association for Supportive Care in Cancer risk index: a multinational scoring system for identifying low-risk febrile neutropenic cancer patients. J Clin Oncol 2000; 18: 3038-51.

9.- Santolaya ME, Álvarez A, Becker A, Cofré J, Enríquez N, O’Ryan $M$, et al: Prospective, multicenter evaluation of risk factors associated with invasive bacterial infection in children with cancer, neutropenia and fever. J Clin Oncol 2001; 19: 3415-21.

10.- Santolaya ME, Álvarez AM, Avilés CL: Tratamiento selectivo de los episodios de neutropenia febril en niños con cáncer. Comité de Infectología, Programa Infantil Nacional de Drogas Antineoplásicas (PINDA). Rev Chil Infect 2004; 21 (3): 213-22.

11.- Santolaya ME, Álvarez AM, Avilés CL, et al: Early hospital discharge followed by outpatient management versus continued hospitalization of children with cancer, fever and neutropenia at low risk for invasive bacterial infection. J Clin Oncol 2004; 22: 3784-9.

12.- Innes HE, Smith DB, O'Reilly SM, et al: Oral antibiotics with early hospital discharge compared with in patient intravenous antibiotics for low-risk febrile neutropenia in patients with cancer: a prospective randomized controlled single centre study. Brit $\mathrm{J}$ of Can 2003; 89: 43-9.

13.- Mullen CA, Buchanan GR: Early hospital discharge of children with cancer treated for fever and neutropenia: identification and management of the low risk patient. J Clin Oncol 1990; 8: 1998-2004.

14.- Holdsworth MT, Hanrahan J, Albanese A, et al: Outpatient management of febrile neutropenia in children with cancer. Pediatr Drugs 2003; 5 (7): 44355.

15.- Klaassen RJ, Goodman TR, Pham B, et al: "Low risk" prediction rule for pediatric oncology patients presenting with fever and neutropenia. J Clin Oncol 2000; 18: 1012-9.

16.- Härtel Ch, Deuster M, Lehrnbecher T, Schultz Ch: Current approaches for risk stratification of infectious complications in pediatric oncology. Pediatr Blood Cancer 2007; 49: 767-73. 